\title{
The effects of two different 8-week stretching protocols on postural stability in amateur sportsmen
}

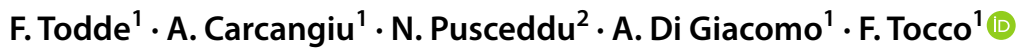

Received: 11 January 2021 / Accepted: 13 September 2021 / Published online: 19 February 2022

(c) The Author(s) 2022

\begin{abstract}
Purpose The purpose of this study was to compare the long-term effects of a static versus proprioceptive neuromuscular facilitation (PNF) stretching protocols by means of spinometry and baropodometry. We hypothesized that PNF may be more effective than static stretching in supporting the static and dynamic balance.

Methods Thirty-six sport science students were divided into three groups: the first group attended a warm-up protocol on the treadmill only (warm-up group) while the other two carried out the same warm-up protocol followed by a static stretching (static group) or by a Contract Relax Antagonist Contract stretching (CRAC group). Stretching programs were performed three times per week on lower limb muscles and the postural stability of each participant was evaluated at entry (pre) and after 8 weeks of training (post).

Results The ellipse area after a warm-up-only protocol did not change; after the static stretching protocol, it was significantly increased (from $111.3 \pm 35.8$ to $135.0 \pm 32.3, p<0.05$ ), while after the CRAC protocol, it significantly decreased (from $119.1 \pm 23.0$ to $88.2 \pm 19.8, p<0.01)$. CRAC group had a significant reduction in average forces $(-3.9 \%$ from pre to post, $p<0.05)$ and in load response $(-0.6 \%$ from pre to post, $p<0.05)$. Pelvic inclination was reduced from 2.22 to $1.33 \mathrm{~mm}$ $(p<0.01)$ and pelvic torsion decreased by $0.94 \pm 0.22^{\circ}(p<0.05)$ after the CRAC protocol.

Conclusions Eight-week CRAC protocol was an excellent training for static and dynamic balance improvement and it was more effective than static stretching.
\end{abstract}

Keywords PNF $\cdot$ CRAC $\cdot$ Balance $\cdot$ Baropodometry $\cdot$ Spinometry

\section{Introduction}

Postural stability is an essential quality for optimal sport performance. A posture that is functional to the sport in question is of utmost importance for the correct management of the training load, be it for the amateur or for the high-level athlete. Posture is definable as a state of muscular-skeletal balance, protecting the supporting structures of the body against injury or progressive deformity. It is the result of interactions between the muscular-skeletal system with afferent and efferent pathways of the central nervous system (CNS) [1]. The proprioceptive system is involved in the

F. Tocco

filippo.tocco@tiscali.it

1 Department of Medical Sciences and Public Health, School of Sport Medicine, University of Cagliari, Cagliari, Italy

2 Ortopedia Chessa S.R.L, Cagliari, Italy maintenance of an effective posture, continuously informing the CNS about the muscle and tendon's lengthening and shortening and at the same time about the joint's position in static and dynamic circumstances [2].

Various studies have demonstrated that having a good postural stability is correlated to a decreased susceptibility to injury. Tropp et al. [3] found that amongst football players those who had balance faults risked incurring an ankle injury 4 times more than those without. McGuine et al. [4] concluded that amongst young basketball players those who scored low on the Gravity Center Test on one leg suffered 7 times the amount of sprains in comparison to those who scored high on the same test.

Static stretching (SS) became really successful in the $70 \mathrm{~s}$ thanks to Bob Anderson, who took inspiration from the stretching methods developed in the practice of yoga, where the range of motion (ROM) was improved using a relaxed lengthening of the muscles through slow breathing [5]. 
The concept of Proprioceptive Neuromuscular Facilitation (PNF) was developed by Kabat \& Knott [6] to improve the recovery of patients who suffered from paralysis PNF stretching incorporate SS and isometric contractions to enhance joint ROM, with 2 common techniques being Contract Relax (CR) and Contract Relax Antagonist Contract (CRAC) [7].

A vast number of studies have confronted these two kinds of stretching studying different variables such flexibility, power and strength. Chen et al. [8] demonstrated that with both a SSand PNF protocol a statistically relevant improvement in strength and hypertrophy was evident after 8 weeks. However, the findings of Chen et al. were not supported by all stretching studies that investigated static and PNF stretching in the long run. In fact, Konrad et al. found that a 6-week static [9] or PNF [10] stretching program of the calf muscles had no effect on maximum voluntary contraction. Yildirim et al. [11] found out that comparing SS, PNF and Mulligan Stretching in the long run over 4 weeks, the latter two performed better in increasing the ROM.

To the best of our knowledge, an analysis regarding the long-term effects of CRAC and SS on postural stability is lacking. The purpose of our study was to compare the effects of these two different stretching protocols on static and dynamic balance. To study this, two groups of Sports Science Course students from Cagliari University performed a PNF or Static stretching protocol on their lower limb muscles for 8 weeks 3 times per week, always following this order: hamstrings, quads, iliopsoas, and calves.

\section{Materials and methods}

\section{Subjects}

Thirty-six voluntary subjects were included in the study. They were Sports Science students at the University of Cagliari (26 men and 10 women; age: $24.27 \pm 4.87$ years, height: $169.25 \pm 10.97 \mathrm{~cm}$ and BMI: $23.59 \pm 2.54$ ). All of them were in good health and none of them had experienced injuries in the previous 6 months. The study was approved by the local ethics committee and was carried out in accordance with The Helsinki Declaration. Written informed consent was obtained before the subjects entered the study.

\section{Experimental design}

Participants were divided into 3 groups of 12: one group (warm-up) attended a warm-up running on the treadmill at a constant speed of $10 \mathrm{~km} / \mathrm{h}$ and $1 \%$ of slope for $10 \mathrm{~min}$, while the other two groups performed the same running program followed by an SS protocol (SS) or by a CRAC protocol (CRAC).
CRAC protocol: (1) Stretch targeted muscle (e.g. hamstrings) to the limit of normal range of motion. Contract the targeted muscle group for 5-10 s while a partner applies sufficient resistance to inhibit movement of the muscle group; (2) relax the targeted muscle group for 3-5 s; (3) contract the antagonist muscle (e.g. quadriceps) for 5-10 s; (4) after a brief period of relaxation, passively stretch the targeted muscle group by applying a controlled, deeper stretch for about 20-30 s into a greater range of motion; (5) relax the muscle for approximately $30 \mathrm{~s}$ before repeating the above process 3 more times. The cycle is repeated for 4 times for each side for the muscle groups selected, always following this order: hamstrings, quads, iliopsoas, and calves.

SS protocol. $30 \mathrm{~s}$ of pain-free stretching, repeated 4 times on the previously cited muscle groups. Training programs were performed three times per week and the postural stability of each participant was evaluated at entry (pre) and after 8 weeks of training (post).

\section{Measurement}

The postural stability of each subject was evaluated pre and post treatment by means of spinometry and baropodometry. The sample size calculation was carried out by a $G^{*}$ Power 3 software and based on the parameters $\alpha=0.05$ (2-sided), power: $1-\beta=0.8$, number of groups $=3$, number of measures $=5$ [12]. To ensure a high scientific standard, all measurements were undertaken by the same expert investigator. Pre- and post-training tests were executed at the same time of day, and the temperature in the laboratory was kept constant at around $20{ }^{\circ} \mathrm{C}$. Students attended the 3 -year course and they had all acquired excellent experience in stretching practices at the time of trial.

Spine rasterstereography (Spine-O-Meter) is a noninvasive test comparable to radiography used for investigating the position of spine, hips and lower limbs [13, 14]. Spinometry utilizes infrared light on the back of the patient. The machine receives the data through reflecting markers and automatically finds the landmarks on the spine: the vertebra $\mathrm{C} 7$, the sacrum and the lumbar dimples. The spine analysis is carried out on the frontal plane (to evaluate the grade of scoliosis) and on the sagittal plane (to evaluate the grade of kyphosis and lordosis). The Formetric $4 \mathrm{D}+$ spinometry uses 10.000 measuring points to conduct a complete morphological test through a triangulation process applied to the videoraster stereography. Spinometry shows up eventual rotational anomalies and non-physiological hip deviations. The system gives an objective evaluation as it allocates numerical values that measure the studied pathology. For the measurements, the subjects were asked to stand freely in their normal postures, facing a black background and with their backs to the camera. Their backs were unclothed and their hair was tied up to provide exposure to the neck. 
The baropodometric exam was performed by a Zebris treadmill system [15]. It can be executed in static or dynamic mode (defined as Gait Analysis). The baropodometric exam consists of the detection of the main pressure points in the foot through the use of sensors disseminated on the treadmill surface. This feature allows the machine to evaluate the altered pressure points in a subject who suffers from flat feet, pronated feet, supinated feet, club foot or altered gait patterns.

\section{Data analysis}

For the baropodometric measurements during the static analysis in the stance phase we analyzed the ellipse area in $\mathrm{mm}^{2}$ (Ellipse) and the pressure difference (\%) between the right and the left foot (average forces). For the gait analysis in the stance phase, we considered the difference between the distribution of the force of left and right foot during the loading response phase of the gait cycle (load response). With regard to the rasterstereographic measurements we considered two parameters linked to posture changes: pelvic inclination and pelvic torsion.

\section{Statistical analysis}

The normality assumption was checked using the Kolmogorov-Smirnov test. Comparisons between tests were performed using the repeated-measures analysis of variance (ANOVA) followed by Newman-Keuls post hoc when appropriate. Differences between groups were analyzed by means of the two-way ANOVA for repeated-measures followed by Tukey post hoc when appropriate. The conditions were the three groups (warm-up only, static and CRAC) and the time points (pre and post). Inter-group time related differences (mean $\pm \mathrm{SD}$ ) were shown as delta $(\Delta)$ values between pre and post and assessed by means of one-way ANOVA followed by Tukey post hoc when appropriate. Statistics were produced utilizing commercially available software (Graph-Pad, Prism). The $\alpha$ level was set at $p<0.05$ in all cases.

\section{Results}

The three protocols were completed by all students and no symptoms or injuries during or following were reported. Figure 1 displays as ellipse area after a warm-up protocol did not change, while after the SS protocol, it significantly increased (from $111.3 \pm 35.8$ to $135.0 \pm 32.3, p<0.02$ ) and

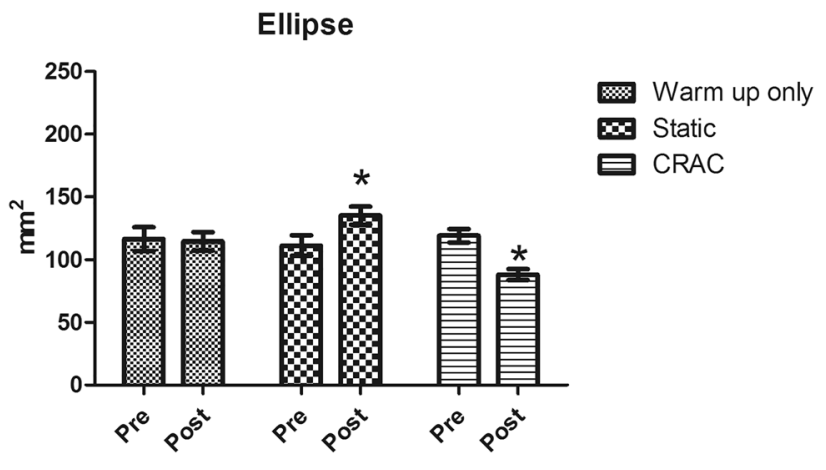

Fig. 1 Ellipse variation after 8 weeks in the three groups. $* p<0.05$ vs. pre

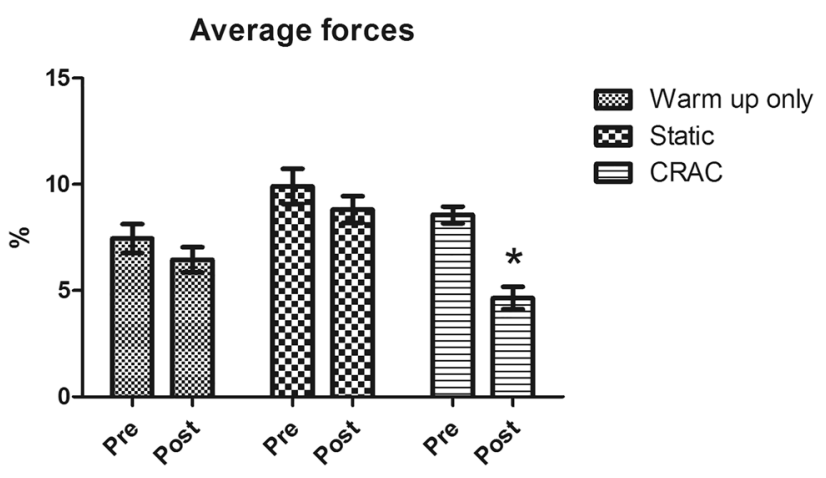

Fig. 2 Average forces variation after 8 weeks in the three groups. $* p<0.05$ vs. pre

after the CRAC protocol significantly decreased (from $119.1 \pm 23.0$ to $88.2 \pm 19.8, p<0.001)$. The overall effect size was $F=3.85$. Effect size $d=0.637$.

Figure 2 shows how the CRAC group had a significant reduction in the difference between average forces $(-3.9 \%$ from pre to post, $p<0.03)$. The overall effect size was $F=11.59$.

Only after CRAC protocol was the difference load response between left and right foot (loading response phase of the gait cycle) significantly reduced $(-0.6 \%$ from pre to post, $p<0.0036$, Fig. 3). The overall effect size was $F=8.84$. Effect size $d=0.965$.

No significant difference in pelvic inclination was found after warm-up and static protocols while it was reduced from 2.22 to $1.33 \mathrm{~mm}(p<0.0086)$ after the CRAC protocol (Fig. 4). The overall effect size was $F=4.96$. Effect size $d=0.723$.

Figure 5 demonstrates that there was no difference in pelvic torsion after warm-up only protocol and SS groups while in the CRAC group difference between pelvic torsion decreased by $0.94 \pm 0.22^{\circ}(p<0.044)$ with an overall effect size of $F=3.21$. Effect size $d=0.581$. 


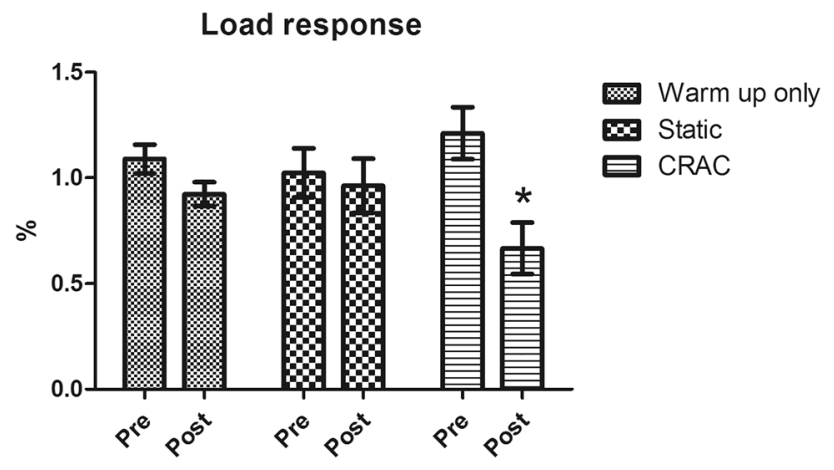

Fig. 3 Load response variation after 8 weeks in the three groups. $* p<0.05$ vs. pre

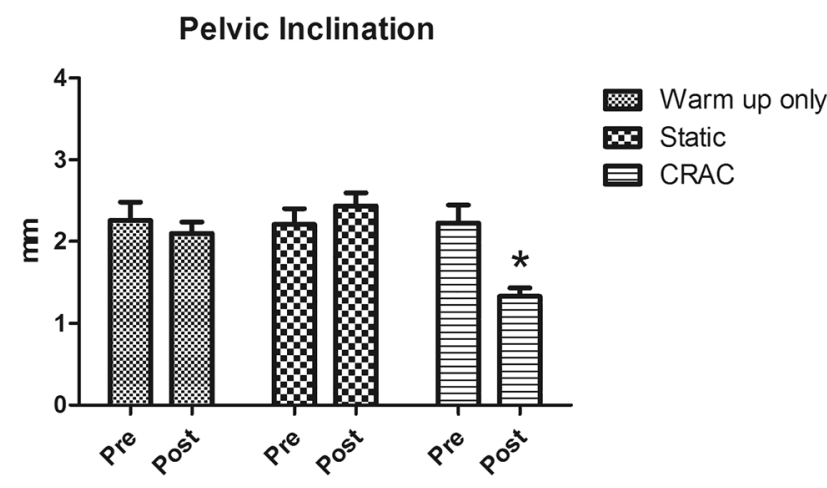

Fig. 4 Pelvic inclination variation after 8 weeks in the three groups. ${ }^{*} p<0.05$ vs. pre

\section{Pelvic Torsion}

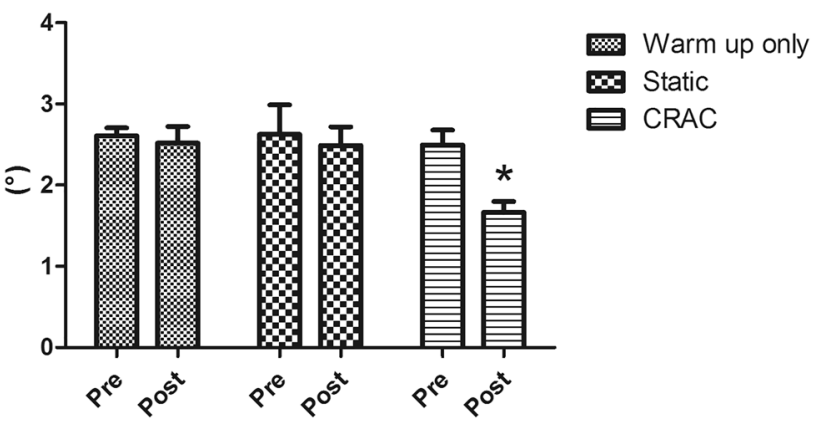

Fig. 5 Pelvic torsion variation after 8 weeks in the three groups. $* p<0.05$ vs. pre

\section{Discussion}

The novelty of this research was to evaluate the effects on postural stability of 2 different stretching protocols lasting 8 weeks. Until today, no other published studies have measured the effects of the CRAC and SS on postural stability combining two different methods such as spinometry and baropodometry.

Concerning the baropodometry tests a first relevant result has been the significant reduction of the ellipse area found in the CRAC group. Thus, the latter showed an improved postural stability in static conditions and this result is in agreement with previous research that (although executed with different methods) described an improvement on mediumlateral stability in the subjects who underwent a CRAC protocol [16]. Conversely, after the SS protocol (by far the most applied in sports), the studied group displayed a decrease of their postural static stability. These results should lead us to reflect on the habitual use of the SS in the warm-up phase of almost every sport. Moreover, in our experiment the dynamic tests on treadmill revealed a re-distribution of the forces between the left and right leg during the stance phase of the gait cycle, which was more effective after the CRAC protocol than others. Thus, the effect of CRAC protocol could be a valid tool for the balance improvement and maintenance in various sports. An improvement in dynamic postural stability could theoretically translate into both an improvement in performance and a lower susceptibility to injury.

With regard to spinometry, in our tests both pelvic inclination and torsion were reduced after CRAC attesting an adjustment towards a more neutral location of the pelvis. An interesting research from a Korean author on the relationship between pelvis stability, posture and gait ability concluded that, for correct posture, the normal location and stability of the pelvis are important and could be helpful to improve balance and gaits [17]. The same author reported that dynamic exercises using PNF patterns as a type of stabilization exercise may be recommended for postural correction for students and workers who have time and place limitations for engaging in exercises [18]. Thus, taking together our results from spinometry and baropodometry tests, PNF stretching seems more effective than SS in preserving the static and dynamic balance. A possible mechanism that could explain our results comes from the research of Minshull and coll. $[19,20]$. They found a lesser impairment of EMD (electromechanical delay, i.e. a component of neuromuscular performance that contributes to the timely, forceful and precise regulation of motor activation) performance following PNF compared to SS conditioning. They conclude that given the importance of rapid muscle activation in the maintenance of dynamic joint stability, PNF conditioning should be the preferred mode of flexibility training over passive for the recreational, rehabilitating, and/or elite athlete involved in explosive performance activities. Thus, our CRAC protocol could be a useful tool in exercise and rehabilitation sessions. Indeed, many authors have reported how PNF-CRAC techniques can be an effective tool in rehabilitation programs $[21,22]$. 


\section{Conclusions}

In our experiment, CRAC protocol proved to be an excellent training for static and dynamic balance improvement and maintenance, and was more effective in comparison to SS. Static and dynamic balance improvement by CRAC training protocols could be of utmost importance if applied to older and sedentary populations. In the sports field, the use of stretching protocols should be rational. The prolonged effects of the different stretching methods have to be carefully studied on a larger scale and on different sports, and further research on this topic is highly desirable.

Acknowledgements The authors wish to thank Joanne Louise Woolgar for his editorial assistance.

Funding Open access funding provided by Università degli Studi di Cagliari within the CRUI-CARE Agreement. No funding was received for this study.

\section{Declarations}

Conflict of interest The authors report no conflict of interest.

Ethical approval The study was approved by the local ethics committee and was carried out in accordance with The Helsinki Declaration.

Informed consent Written informed consent was obtained before the subjects entered the study.

Open Access This article is licensed under a Creative Commons Attribution 4.0 International License, which permits use, sharing, adaptation, distribution and reproduction in any medium or format, as long as you give appropriate credit to the original author(s) and the source, provide a link to the Creative Commons licence, and indicate if changes were made. The images or other third party material in this article are included in the article's Creative Commons licence, unless indicated otherwise in a credit line to the material. If material is not included in the article's Creative Commons licence and your intended use is not permitted by statutory regulation or exceeds the permitted use, you will need to obtain permission directly from the copyright holder. To view a copy of this licence, visit http://creativecommons.org/licenses/by/4.0/.

\section{References}

1. Carini F, Mazzola M, Fici C, Palmeri S, Messina M, Damiani P, Tomasello G (2017) Posture and posturology, anatomical and physiological profiles: overview and current state of art. Acta Biomed 88(1):11-16

2. Proske U, Gandevia SC (2012) The proprioceptive senses: their roles in signaling body shape, body position and movement, and muscle force. Physiol Rev 92:1651-1697

3. Tropp H, Ekstrand J, Gillquist J (1984) Stabilometry in functional instability of the ankle and its value in predicting injury. Med Sci Sports Exerc 16(1):64-66

4. McGuine TA, Greene JJ, Best T, Leverson G (2000) Balance as a predictor of ankle injuries in high school basketball players. Clin J Sports Med Off J Can Acad Sports Med 10(4):239-244
5. Anderson B, Burke ER (1991) Scientific, medical, and practical aspects of stretching. Clin Sports Med 10(1):63-86

6. Kabat H, Knott M (1953) Proprioceptive facilitation technics for treatment of paralysis. Phys Ther Rev 33(2):53-64

7. Sharman MJ, Cresswell AG, Riek S (2006) Proprioceptive neuromuscular facilitation stretching : mechanisms and clinical implications. Sports Med 36(11):929-939

8. Chen C-H, Chen TC, Chen H-L, Lin M-J, Wu C-J, Tseng K-W (2009) Effects of 8-week static stretch and PNF training on the angle-torque relationship. J Med Biol Eng 29(4):196-201

9. Konrad A, Tilp M (2014) Increased range of motion after static stretching is not due to changes in muscle and tendon structures. Clin Biomech (Bristol, Avon) 29(6):636-642

10. Konrad A, Gad M, Tilp M (2015) Effect of PNF stretching training on the properties of human muscle and tendon structures. Scand J Med Sci Sports 25(3):346-355

11. Yıldırım MS, Ozyurek S, Tosun OC, Uzer S, Gelecek N (2016) Comparison of effects of static, proprioceptive neuromuscular facilitation and Mulligan stretching on hip flexion range of motion: a randomized controlled trial. Biol Sport 33(1):89-94

12. Faul F, Erdfelder E, Lang AG, Buchner A (2007) G*Power 3: A flexible statistical power analysis program for the social, behavioral, and biomedical sciences. Behav Res Methods 39:175-191

13. Frerich JM, Hertzler K, Knott P, Mardjetko S (2012) Comparison of radiographic and surface topography measurements in adolescents with idiopathic scoliosis. Open Orthop J 6:261-265

14. Mohokum M, Schülein S, Skwara A (2015) The validity of rasterstereography: a systematic review. Orthop Rev (Pavia) 7(3):5899

15. Reed LF, Urry SR, Wearing SC (2013) Reliability of spatiotemporal and kinetic gait parameters determined by a new instrumented treadmill system. BMC Musculoskelet Disord 21(14):249

16. Ryan EE, Rossi MD, Lopez R (2010) The effects of the contractrelax-antagonist contract form of proprioceptive neuromuscular facilitation stretching on 254 postural stability. J Strength Cond Res 24(7):1888-1894

17. Cho M (2013) The influence of pelvic adjustment on the posture of female university students. J Phys Ther Sci 25:785-787

18. Cho M, Gong W (2017) The effects of dynamic exercise using the proprioceptive neuromuscular facilitation pattern on posture in healthy adults. J Phys Ther Sci 29(6):1070-1073

19. Minshull C, Rees D, Gleeson NP (2011) Joint angle affects volitional and magnetically-evoked neuromuscular performance differentially. J Electromyogr Kinesiol 21(4):672-677

20. Minshull C, Eston R, Bailey A, Rees D, Gleeson N (2014) The differential effects of PNF versus passive stretch conditioning on neuromuscular performance. Eur J Sport Sci 14:233-241

21. González-Ravé JM, Sánchez-Gómez A, Santos-García DJ (2012) Efficacy of two different stretch training programs (passive vs. proprioceptive neuromuscular facilitation) on shoulder and hip range of motion in older people. J Strength Cond Res 26(4):1045-1051

22. Guiu-Tula FX, Cabanas-Valdés R, Sitjà-Rabert M, Urrútia G, Gómara-Toldrà N (2017) The Efficacy of the proprioceptive neuromuscular facilitation (PNF) approach in stroke rehabilitation to improve basic activities of daily living and quality of life: a systematic review and meta-analysis protocol. BMJ Open 7(12): 016739

Publisher's Note Springer Nature remains neutral with regard to jurisdictional claims in published maps and institutional affiliations. 